\title{
ExploreNEOs. III. PHYSICAL CHARACTERIZATION OF 65 POTENTIAL SPACECRAFT TARGET ASTEROIDS
}

\author{
Michael Mueller $^{1}$, M. Delbo' ${ }^{1}$, J. L. Hora ${ }^{2}$, D. E. Trilling ${ }^{3}$, B. Bhattacharya ${ }^{4}$, W. F. Bottke ${ }^{5}$, S. Chesley ${ }^{6}$, \\ J. P. Emeri ${ }^{7}$, G. Fazio ${ }^{2}$, A. W. Harris ${ }^{8}$, A. Mainzer ${ }^{6}$, M. Mommert ${ }^{8}$, B. Penprase ${ }^{9}$, H. A. Smith ${ }^{2}$, T. B. Spahr ${ }^{2}$, \\ J. A. Stansberry ${ }^{10}$, AND C. A. Thomas ${ }^{3}$ \\ ${ }^{1}$ UNS-CNRS-Observatoire de la Côte d'Azur, Laboratoire Cassiopée, BP 4229, 06304 Nice Cedex 04, France; michael.mueller@oca.eu \\ ${ }^{2}$ Harvard-Smithsonian Center for Astrophysics, 60 Garden Street, MS-65, Cambridge, MA 02138, USA \\ ${ }^{3}$ Department of Physics and Astronomy, Northern Arizona University, Flagstaff, AZ 86001, USA \\ ${ }^{4}$ NASA Herschel Science Center, Caltech, M/S 100-22, 770 South Wilson Avenue, Pasadena, CA 91125, USA \\ ${ }^{5}$ Southwest Research Institute, 1050 Walnut Street, Suite 300, Boulder, CO 80302, USA \\ ${ }^{6}$ Jet Propulsion Laboratory, California Institute of Technology, Pasadena, CA 91109, USA \\ ${ }^{7}$ Department of Earth and Planetary Sciences, University of Tennessee, 1412 Circle Dr., Knoxville, TN 37996, USA \\ ${ }^{8}$ DLR Institute of Planetary Research, Rutherfordstrasse 2, 12489 Berlin, Germany \\ ${ }^{9}$ Department of Physics and Astronomy, Pomona College, 610 N. College Avenue, Claremont, CA 91711, USA \\ ${ }^{10}$ Steward Observatory, University of Arizona, 933 North Cherry Avenue, Tucson, AZ 85721, USA \\ Received 2010 September 22; accepted 2011 January 25; published 2011 February 22
}

\begin{abstract}
Space missions to near-Earth objects (NEOs) are being planned at all major space agencies, and recently a manned mission to an NEO was announced as a NASA goal. Efforts to find and select suitable targets (plus backup targets) are severely hampered by our lack of knowledge of the physical properties of dynamically favorable NEOs. In particular, current mission scenarios tend to favor primitive low-albedo objects. For the vast majority of NEOs, the albedo is unknown. Here we report new constraints on the size and albedo of 65 NEOs with rendezvous $\Delta v<7 \mathrm{~km} \mathrm{~s}^{-1}$. Our results are based on thermal-IR flux data obtained in the framework of our ongoing (2009-2011) ExploreNEOs survey using NASA's "Warm-Spitzer" space telescope. As of 2010 July 14, we have results for 293 objects in hand (including the 65 low- $\Delta v$ NEOs presented here); before the end of 2011, we expect to have measured the size and albedo of $\sim 700$ NEOs (including probably $\sim 160$ low- $\Delta v$ NEOs). While there are reasons to believe that primitive volatile-rich materials are universally low in albedo, the converse need not be true: the orbital evolution of some dark objects likely has caused them to lose their volatiles by coming too close to the Sun. For all our targets, we give the closest perihelion distance they are likely to have reached (using orbital integrations from Marchi et al. 2009) and corresponding upper limits on the past surface temperature. Low- $\Delta v$ objects for which both albedo and thermal history may suggest a primitive composition include (162998) 2001 SK162, (68372) 2001 PM9, and (100085) 1992 UY4.
\end{abstract}

Key words: infrared: planetary systems - minor planets, asteroids: general - radiation mechanisms: thermal space vehicles - surveys

\section{INTRODUCTION}

To date, two near-Earth objects (NEOs) have been targeted by space missions, both yielding a wealth of fascinating and groundbreaking insights into the past and current state of the solar system: NASA's NEAR-Shoemaker mission went into orbit around its target (433) Eros in 2000 and landed on it in the following year; the Japanese mission Hayabusa arrived at (25143) Itokawa in 2005 and scrutinized the NEO for a few months. In 2010 June, Hayabusa succeeded in returning asteroid dust samples to Earth.

Given the remarkable success of these missions, it is perhaps not surprising that robotic NEO mission concepts are being considered at space agencies across the planet, including NASA's OSIRIS-REx, JAXA's Hayabusa 2, and ESA's Marco Polo (see, e.g., Lauretta et al. 2010; Okada et al. 2010; Michel et al. 2009 for recent updates on these missions). In a speech in 2010 April, President Obama announced the goal of a manned space mission to an asteroid (see Abell et al. 2009 for a corresponding NASA mission scenario including robotic precursor missions).

Finding a suitable target asteroid is one of the challenging aspects of mission planning. Targets are tightly constrained in terms of their orbital dynamics and physical properties. Furthermore, launch windows are usually tight and the planning process long. Unforeseen delays due to technological or finan- cial problems risk eliminating the nominal target; it is therefore generally advisable to plan for contingency or backup targets.

Dynamics, $\Delta v$. As discussed by Shoemaker \& Helin (1978), mission cost depends chiefly on the required amount of propellant, which follows from the total specific linear momentum, $\Delta v$, that must be imparted on the spacecraft for it to reach the target orbit. Minimizing $\Delta v$ is therefore a top priority for practical reasons. It is worth emphasizing that there are a large number of NEOs that are reachable at a lower $\Delta v$ than that required to reach Mars.

A realistic assessment of $\Delta v$ depends on the specific mission scenario and timing and must be evaluated on a target-bytarget basis. A customary first-order estimate is the $\Delta v$ of a Hohmann transfer orbit, which is an analytic function of the orbital elements (see Shoemaker \& Helin 1978). Thus, in a first target selection process objects with sufficiently small "Hohmann- $\Delta v$ " are identified. Only those objects need to be studied in detail. For the purposes of this study, we will refer to the "Hohmann- $\Delta v$ " as $\Delta v$ (without qualifiers).

Physical properties. For the vast majority of NEOs, including low- $\Delta v$ objects, nothing is known about their physical properties. Frequently, however, mission concepts require the target to be within a given size or mass range, e.g., in order to enable the spacecraft to orbit the target. Moreover, the science goals of some current mission scenarios require their target to be a 
"primitive" object, translating into constraints on their albedo and thermal history (see below).

Primitive objects. Some meteorites contain surprisingly pristine material that has suffered very little modification since the early days of the solar system. Their asteroidal parent bodies are of particular interest for some NEO missions (especially since both NEAR-Shoemaker and Hayabusa targeted S-type asteroids, which have undergone significant processing).

Judging from meteorite analogs, asteroids with very low albedo (geometric albedo $p_{\mathrm{V}} \lesssim 7.5 \%$ ) are very likely to be "primitive" and vice versa (Fernández et al. 2005). A word of caution applies to NEOs, however: their relative proximity to the Sun can potentially cause their surfaces to heat up to the point that thermal surface alterations occur (devolatilization, chemical reactions, etc.). There are hence two necessary conditions for an NEO to have a primitive surface: a low albedo and an orbital history that never brought the perihelion too close to the Sun.

ExploreNEOs. This is the third paper describing results from the ongoing (2009-2011) ExploreNEOs survey (following Trilling et al. 2010; Harris et al. 2011). The primary goal of this survey is to measure the size and albedo of $\sim 700$ NEOs based on observations with NASA's "Warm-Spitzer" space telescope. Trilling et al. (2010) describe the goals and methods of the survey along with results for the first 101 NEOs; Harris et al. (2011) check the accuracy of the ExploreNEOs results against values published in the literature (where available) and find diameters to be typically consistent within $20 \%$, albedos within $50 \%$.

As of 2010 July 14, we have data for 293 NEOs in hand including 65 objects with $\Delta v<7 \mathrm{~km} \mathrm{~s}^{-1}$. By the end of the survey, i.e., before the end of 2011, we expect to have measured $\sim 160$ low- $\Delta v$ objects. We chose to publish this first batch of results in order to alert the community to the existence of our growing database of characterized low- $\Delta v$ objects.

Overview of this work. In Section 2, we describe our photometric data. Our modeling approach is described in Section 3, and resulting diameter and albedo estimates are presented in Section 4. In Section 5, we study the thermal history of our targets. We discuss the implications of our results in Section 6 and summarize our conclusions in Section 7.

\section{WARM-SPITZER OBSERVATIONS}

The observations reported herein use the post-cryogenic ("warm") mode of the IRAC camera (Fazio et al. 2004) on board the Spitzer Space Telescope (Werner et al. 2004). Each NEO target is observed in the two photometric channels (channels 1 and 2) with central wavelengths of around 3.6 and $4.5 \mu \mathrm{m}$, respectively. Observations are built up from frames that alternate repeatedly between the two channels, such that the resulting fluxes are quasi-simultaneous. Further details on our observation design and data reduction are given in Trilling et al. (2010).

In this work, we restrict ourselves to objects observed on or before 2010 July 14 with a rendezvous $\Delta v \leqslant 7 \mathrm{~km} \mathrm{~s}^{-1}$. $\Delta v$ values are taken from Lance Benner's online list of $\Delta v$ for all NEOs, ${ }^{11}$ which is calculated from the orbital elements and the Shoemaker \& Helin (1978) formalism.

In Table 1, we present the measured in-band fluxes and the observing circumstances as taken from JPL's Horizons ephemeris server. $H$ magnitudes are assumed to be uncertain by

\footnotetext{
11 http://echo.jpl.nasa.gov/ lance/delta_v/delta_v.rendezvous.html
}

0.5 mag (see Section 3). Observations carried out on or before 2009 November 4 have been presented in Trilling et al. (2010), later observations are new here.

\section{THERMAL MODELING}

We use an updated version of the thermal-modeling pipeline used in previous publications resulting from the ExploreNEOs survey (Trilling et al. 2010; Harris et al. 2011). For completeness, we briefly summarize the more detailed description given in Trilling et al. (2010). Section 3.1 presents the updates relative to the previous pipeline, chiefly an estimation of the statistical uncertainty of our results. Due to said update, some of our results differ slightly (but within the error bars) from the preliminary results given in Trilling et al. (2010). A reanalysis of our entire data set is deferred to a later work.

NEO fluxes at Warm-Spitzer wavelengths have significant contributions from reflected sunlight and from thermal emission. We are interested in the latter in order to calculate the target size and albedo using a thermal model. Therefore, in a first step, we estimate the amount of reflected solar radiation using the method first described by Mueller et al. (2007), then refined in Trilling et al. (2008, 2010). Briefly, we calculate the expected $V$ magnitude based on the observing circumstances given in Table 1 and extrapolate to 3.6 and $4.5 \mu \mathrm{m}$ fluxes using published values of the solar flux at those wavelengths and the Sun's $V$ magnitude. We also assume the spectral reflectivity at WarmSpitzer wavelengths to be 1.4 times that in the $V$ band (see Trilling et al. 2008, 2010; Harris et al. 2009). In-band thermal flux equals total flux minus reflected flux. In rare cases, the calculated reflected flux exceeds the measured flux, leading to unphysical negative thermal fluxes in channel 1. In these cases (which we expect are due to inaccurate $H$ magnitudes and/or light curve effects), we drop the channel-1 flux from the thermal analysis.

In-band thermal fluxes are color corrected to take account of the spectral breadth of IRAC's filters and the significant difference between the spectral shape of asteroidal thermal emission and the stellar-like spectrum assumed in IRAC flux calibration; color-correction factors for the reflected solar component are negligible. Color-correction factors are calculated for each target using the IRAC passbands given by Carey et al. (2010), the observing circumstances, and a suitable thermal model. As found by Mueller et al. (2007), the dependence of color-correction factors on the physical properties of the asteroid can be neglected.

Diameter and albedo are estimated from the final thermal fluxes using the Near-Earth Asteroid Thermal Model (NEATM) (Harris 1998). The NEATM contains a dimensionless parameter $\eta$ that describes the effective surface temperature. Trilling et al. (2008) found that data quality does not usually allow to fit $\eta$ to Warm-Spitzer data of NEOs, but that reasonable estimates of diameter and albedo can still be obtained by assuming an empirical linear relationship between $\eta$ and solar phase angle $\alpha$. That relationship was established by Delbo' et al. (2003); here we use an updated relationship (based on a slightly larger data set) by Wolters et al. (2008):

$$
\eta=(0.91 \pm 0.17)+(0.013 \pm 0.004) \alpha(\text { in deg }) .
$$

\subsection{Monte Carlo Approach}

In order to provide a realistic estimate of the uncertainty in our diameter and albedo results, we use a Monte Carlo approach in which various sources of uncertainty are considered: 
Table 1

Spitzer Data Sorted by $\Delta v$

\begin{tabular}{|c|c|c|c|c|c|c|c|c|}
\hline $\begin{array}{l}\Delta v \\
\left(\mathrm{~km} \mathrm{~s}^{-1}\right)\end{array}$ & Object & $\begin{array}{l}\text { Time } \\
\text { (UT) }\end{array}$ & $H$ & $\begin{array}{c}r \\
(\mathrm{AU})\end{array}$ & $\begin{array}{c}\Delta \\
(\mathrm{AU})\end{array}$ & $\begin{array}{c}\alpha \\
(\mathrm{deg})\end{array}$ & $\begin{array}{c}\mathrm{f} 36 \\
\text { (mJy) }\end{array}$ & $\begin{array}{c}\mathrm{f} 45 \\
(\mathrm{mJy})\end{array}$ \\
\hline 4.632 & (25143) Itokawa & 2010 May 15 14:45:09 & 19.20 & 1.018 & 0.052 & 74.56 & $1.767 \pm 0.041$ & $6.033 \pm 0.072$ \\
\hline 4.755 & 1996 XВ27 & 2010 Jul 12 06:41:36 & 21.84 & 1.121 & 0.192 & 51.92 & $0.0178 \pm 0.0040$ & $0.0428 \pm 0.0061$ \\
\hline 4.887 & (10302) $1989 \mathrm{ML}$ & 2009 Aug 21 03:09:35 & 19.50 & 1.100 & 0.152 & 56.01 & $0.229 \pm 0.015$ & $0.560 \pm 0.022$ \\
\hline 5.276 & (99799) 2002 LJ3 & 2009 Sep 19 22:38:44 & 18.10 & 1.238 & 0.354 & 46.18 & $0.125 \pm 0.015$ & $0.392 \pm 0.020$ \\
\hline 5.280 & 2001 CQ36 & 2010 Apr 15 13:27:01 & 22.45 & 1.069 & 0.125 & 55.50 & $0.0215 \pm 0.0044$ & $0.0787 \pm 0.0081$ \\
\hline 5.302 & (52381) 1993 HA & 2009 Nov 18 12:09:41 & 20.20 & 1.257 & 0.402 & 46.59 & $0.0303 \pm 0.0052$ & $0.150 \pm 0.011$ \\
\hline 5.328 & 2000 YF29 & 2010 Feb 20 04:43:34 & 20.16 & 1.015 & 0.123 & 83.03 & $0.131 \pm 0.012$ & $0.467 \pm 0.020$ \\
\hline 5.391 & (1943) Anteros & 2009 Sep 15 00:09:47 & 15.75 & 1.548 & 0.951 & 40.17 & $0.189 \pm 0.013$ & $0.582 \pm 0.022$ \\
\hline 5.486 & (138911) 2001 AE2 & 2009 Aug 13 14:53:36 & 19.10 & 1.330 & 0.483 & 41.78 & $0.0244 \pm 0.0047$ & $0.0888 \pm 0.0086$ \\
\hline 5.487 & 2006 SY 5 & 2009 Jul 30 18:18:27 & 22.08 & 1.093 & 0.136 & 54.07 & $0.0312 \pm 0.0051$ & $0.1167 \pm 0.0097$ \\
\hline 5.555 & (162416) 2000 EH26 & 2010 Apr 29 07:39:49 & 21.70 & 1.125 & 0.217 & 51.21 & $0.0378 \pm 0.0058$ & $0.1181 \pm 0.0099$ \\
\hline 5.565 & (162998) 2001 SK162 & 2009 Dec 17 23:35:37 & 18.00 & 1.135 & 0.451 & 63.68 & $0.815 \pm 0.028$ & $3.184 \pm 0.051$ \\
\hline 5.653 & (68372) 2001 PM9 & 2009 Aug 20 10:32:54 & 18.90 & 1.130 & 0.204 & 53.62 & $3.928 \pm 0.059$ & $19.61 \pm 0.14$ \\
\hline 5.719 & (12923) Zephyr & 2010 May 21 04:54:42 & 16.10 & 1.477 & 0.876 & 41.50 & $0.253 \pm 0.021$ & $0.453 \pm 0.023$ \\
\hline 6.041 & (85938) 1999 DJ4 & 2009 Aug 13 15:40:17 & 18.60 & 1.409 & 0.688 & 43.10 & $0.0172 \pm 0.0039$ & $0.0620 \pm 0.0072$ \\
\hline 6.069 & (433) Eros & 2009 Aug 25 06:16:47 & 11.16 & 1.702 & 1.218 & 36.50 & $13.99 \pm 0.11$ & $40.23 \pm 0.20$ \\
\hline 6.070 & 2000 GV147 & 2009 Oct 24 06:09:17 & 19.03 & 1.064 & 0.336 & 74.34 & $0.0998 \pm 0.010$ & $0.337 \pm 0.017$ \\
\hline 6.086 & 2000 XK44 & 2010 Jan 11 03:12:17 & 17.73 & 1.288 & 0.848 & 51.85 & $0.0285 \pm 0.0051$ & $0.1040 \pm 0.0094$ \\
\hline 6.106 & 1993 RA & 2009 Nov 17 02:57:14 & 18.91 & 1.191 & 0.587 & 59.20 & $0.0165 \pm 0.0039$ & $0.0544 \pm 0.0068$ \\
\hline 6.130 & (177614) 2004 HK33 & 2009 Jul 30 16:25:59 & 17.60 & 1.057 & 0.095 & 63.88 & $5.895 \pm 0.071$ & $22.92 \pm 0.14$ \\
\hline 6.191 & $1998 \mathrm{VO}$ & 2009 Dec 13 03:44:37 & 20.37 & 1.024 & 0.021 & 76.62 & $4.481 \pm 0.063$ & $15.31 \pm 0.11$ \\
\hline 6.196 & 2006 SV19 & 2009 Sep 15 16:08:48 & 17.76 & 1.166 & 0.672 & 60.97 & $0.109 \pm 0.010$ & $0.435 \pm 0.019$ \\
\hline 6.236 & 2005 JA22 & 2009 Nov 10 03:50:52 & 18.47 & 1.296 & 0.489 & 46.66 & $0.0908 \pm 0.0093$ & $0.319 \pm 0.017$ \\
\hline 6.240 & (1627) Ivar & 2010 Jun 16 08:39:40 & 13.20 & 1.933 & 1.249 & 27.62 & $1.264 \pm 0.033$ & $2.670 \pm 0.048$ \\
\hline 6.279 & (87024) 2000 JS66 & 2010 Jan 10 19:24:49 & 18.70 & 1.104 & 0.567 & 65.82 & $0.0163 \pm 0.0023$ & $0.0427 \pm 0.0061$ \\
\hline 6.323 & (22099) 2000 EX106 & 2010 Jan 11 09:47:46 & 18.00 & 1.029 & 0.246 & 79.60 & $0.225 \pm 0.015$ & $0.842 \pm 0.027$ \\
\hline 6.364 & 2003 SL5 & 2009 Sep 19 23:06:19 & 19.14 & 1.114 & 0.164 & 53.62 & $0.289 \pm 0.017$ & $1.052 \pm 0.029$ \\
\hline 6.364 & (35107) $1991 \mathrm{VH}$ & 2010 Mar 20 17:04:49 & 16.90 & 1.172 & 0.660 & 58.78 & $0.113 \pm 0.011$ & $0.477 \pm 0.021$ \\
\hline 6.379 & (172974) 2005 YW55 & 2010 Mar 24 09:16:52 & 19.30 & 1.242 & 0.386 & 44.41 & $0.0615 \pm 0.0078$ & $0.171 \pm 0.013$ \\
\hline 6.405 & (65679) 1989 UQ & 2009 Oct 15 20:50:49 & 19.40 & 1.129 & 0.237 & 58.64 & $0.405 \pm 0.061$ & $2.264 \pm 0.043$ \\
\hline 6.431 & (159402) 1999 AP10 & 2009 Aug 31 22:54:55 & 16.40 & 1.292 & 0.838 & 52.32 & $0.090 \pm 0.010$ & $0.278 \pm 0.016$ \\
\hline 6.491 & (143651) 2003 QO104 & 2009 Aug 21 02:40:17 & 16.00 & 1.402 & 0.801 & 45.93 & $0.276 \pm 0.042$ & $1.052 \pm 0.031$ \\
\hline 6.507 & $1989 \mathrm{AZ}$ & 2010 Feb 19 07:05:07 & 19.49 & 1.003 & 0.044 & 93.49 & $8.998 \pm 0.090$ & $46.28 \pm 0.19$ \\
\hline 6.512 & 2006 WO127 & 2009 Nov 04 01:09:24 & 16.18 & 1.528 & 0.889 & 40.23 & $0.102 \pm 0.011$ & $0.350 \pm 0.018$ \\
\hline 6.516 & (140158) 2001 SX169 & 2010 Jan 26 04:30:24 & 18.30 & 1.253 & 0.430 & 47.17 & $0.092 \pm 0.010$ & $0.362 \pm 0.018$ \\
\hline 6.526 & (100085) 1992 UY4 & 2010 Feb 07 01:04:57 & 17.80 & 1.226 & 0.832 & 54.76 & $0.317 \pm 0.017$ & $1.694 \pm 0.037$ \\
\hline 6.534 & (85839) 1998 YO4 & 2010 Apr 05 10:55:52 & 16.30 & 1.326 & 0.783 & 48.87 & $0.179 \pm 0.014$ & $0.792 \pm 0.026$ \\
\hline 6.539 & (68359) 2001 OZ13 & 2010 May 26 02:33:35 & 17.60 & 1.520 & 0.896 & 39.56 & $0.0243 \pm 0.0079$ & $0.0443 \pm 0.0078$ \\
\hline 6.577 & (11398) 1998 YP11 & 2010 Apr 11 12:59:19 & 16.30 & 1.480 & 0.700 & 36.03 & $0.240 \pm 0.016$ & $0.830 \pm 0.027$ \\
\hline 6.608 & (155334) 2006 DZ169 & 2009 Dec 20 23:59:59 & 17.10 & 1.636 & 0.871 & 32.65 & $0.0548 \pm 0.0072$ & $0.148 \pm 0.011$ \\
\hline 6.609 & (175706) 1996 FG3 & 2010 May 02 05:19:51 & 18.20 & 1.213 & 0.418 & 51.02 & $0.853 \pm 0.028$ & $4.391 \pm 0.062$ \\
\hline 6.610 & (52760) 1998 ML14 & 2010 Jan 26 05:06:11 & 17.50 & 1.075 & 0.484 & 69.33 & $0.127 \pm 0.012$ & $0.463 \pm 0.020$ \\
\hline 6.628 & (138947) 2001 BA40 & 2010 Jan 10 17:16:01 & 18.40 & 1.173 & 0.721 & 59.14 & $0.0135 \pm 0.0035$ & $0.0592 \pm 0.0071$ \\
\hline 6.652 & $2002 \mathrm{QE} 7$ & 2009 Sep 23 04:42:21 & 19.33 & 1.217 & 0.329 & 47.87 & $0.0662 \pm 0.0080$ & $0.188 \pm 0.013$ \\
\hline 6.652 & (5626) $1991 \mathrm{FE}$ & 2009 Aug 21 03:23:36 & 14.70 & 1.689 & 0.972 & 33.08 & $0.434 \pm 0.020$ & $1.209 \pm 0.031$ \\
\hline 6.661 & (66251) 1999 GJ2 & 2010 Feb 09 16:33:48 & 17.00 & 1.751 & 1.038 & 30.67 & $0.0294 \pm 0.0052$ & $0.0512 \pm 0.0066$ \\
\hline 6.692 & (85990) 1999 JV6 & 2010 Jul 12 14:35:39 & 20.00 & 1.062 & 0.116 & 62.78 & $0.978 \pm 0.029$ & $4.922 \pm 0.064$ \\
\hline 6.703 & 1998 SE36 & 2010 Apr 28 10:35:18 & 19.32 & 1.294 & 0.457 & 42.22 & $0.0280 \pm 0.0050$ & $0.1089 \pm 0.0096$ \\
\hline 6.738 & (5645) $1990 \mathrm{SP}$ & 2010 Jun 15 07:08:46 & 17.00 & 1.780 & 1.095 & 30.80 & $0.0496 \pm 0.0071$ & $0.237 \pm 0.015$ \\
\hline 6.747 & (3671) Dionysus & 2010 Jun 12 23:01:07 & 16.30 & 1.136 & 0.518 & 62.60 & $0.180 \pm 0.013$ & $0.390 \pm 0.019$ \\
\hline 6.750 & 2002 HF8 & 2009 Aug 24 23:44:37 & 18.27 & 1.262 & 0.449 & 48.69 & $0.1093 \pm 0.0099$ & $0.455 \pm 0.020$ \\
\hline 6.751 & (164202) $2004 \mathrm{EW}$ & 2009 Aug 06 05:32:19 & 20.80 & 1.049 & 0.158 & 75.37 & $0.0459 \pm 0.0062$ & $0.135 \pm 0.010$ \\
\hline 6.757 & $2002 \mathrm{UN} 3$ & 2010 Feb 12 22:31:31 & 18.60 & 1.325 & 0.503 & 41.88 & $0.0343 \pm 0.0058$ & $0.0555 \pm 0.0070$ \\
\hline 6.791 & (90373) 2003 SZ219 & 2009 Dec 20 00:33:14 & 18.80 & 1.313 & 0.466 & 42.42 & $0.0332 \pm 0.0057$ & $0.0686 \pm 0.0078$ \\
\hline 6.817 & (40329) $1999 \mathrm{ML}$ & 2009 Sep 03 00:25:51 & 17.70 & 1.344 & 0.501 & 41.48 & $0.197 \pm 0.014$ & $0.623 \pm 0.023$ \\
\hline 6.828 & (6239) Minos & 2010 Mar 20 17:55:44 & 17.90 & 1.122 & 0.340 & 61.27 & $0.114 \pm 0.012$ & $0.28 \pm 0.17$ \\
\hline 6.830 & 2005 EJ & 2010 Mar 19 13:32:33 & 19.87 & 1.225 & 0.414 & 49.10 & $0.0215 \pm 0.0046$ & $0.0530 \pm 0.0067$ \\
\hline 6.840 & (5646) $1990 \mathrm{TR}$ & 2009 Dec 01 18:48:50 & 14.30 & 1.857 & 1.679 & 33.09 & $0.0393 \pm 0.0063$ & $0.0809 \pm 0.0085$ \\
\hline 6.865 & 2003 WO7 & 2009 Oct 09 15:38:14 & 18.91 & 1.237 & 0.418 & 50.83 & $0.225 \pm 0.022$ & $0.517 \pm 0.024$ \\
\hline 6.867 & 1999 RH33 & 2010 Apr 04 02:25:30 & 19.13 & 1.286 & 0.463 & 43.65 & $0.0177 \pm 0.0042$ & $0.0371 \pm 0.0057$ \\
\hline 6.963 & (10115) $1992 \mathrm{SK}$ & 2010 Apr 13 09:53:12 & 17.00 & 1.170 & 0.307 & 50.53 & $0.549 \pm 0.024$ & $1.896 \pm 0.042$ \\
\hline 6.963 & 2003 BT47 & 2010 Apr 21 05:07:07 & 17.42 & 1.289 & 0.588 & 48.67 & $0.174 \pm 0.013$ & $0.670 \pm 0.024$ \\
\hline 6.974 & (5620) Jasonwheeler & 2009 Aug 25 07:00:34 & 17.00 & 1.321 & 0.620 & 48.68 & $0.258 \pm 0.016$ & $1.252 \pm 0.033$ \\
\hline 6.981 & (152563) $1992 \mathrm{BF}$ & 2010 Apr 03 23:55:21 & 19.80 & 1.125 & 0.236 & 53.50 & $0.282 \pm 0.017$ & $1.377 \pm 0.034$ \\
\hline 6.994 & (138971) 2001 CB21 & 2009 Dec 06 22:09:34 & 18.40 & 1.033 & 0.272 & 79.87 & $0.176 \pm 0.014$ & $0.591 \pm 0.023$ \\
\hline
\end{tabular}

Notes. Times given are roughly mid-observation as measured on Spitzer. $H$ is the absolute optical magnitude (taken from Horizons and assumed to be uncertain by \pm 0.5 mag). $r$ and $\Delta$ are heliocentric and Spitzer-centric distance, respectively, and $\alpha$ is the solar phase angle. f36 and f45 are the flux at $\sim 3.6$ and $4.5 \mu \mathrm{m}$, respectively (channels 1 and 2). 

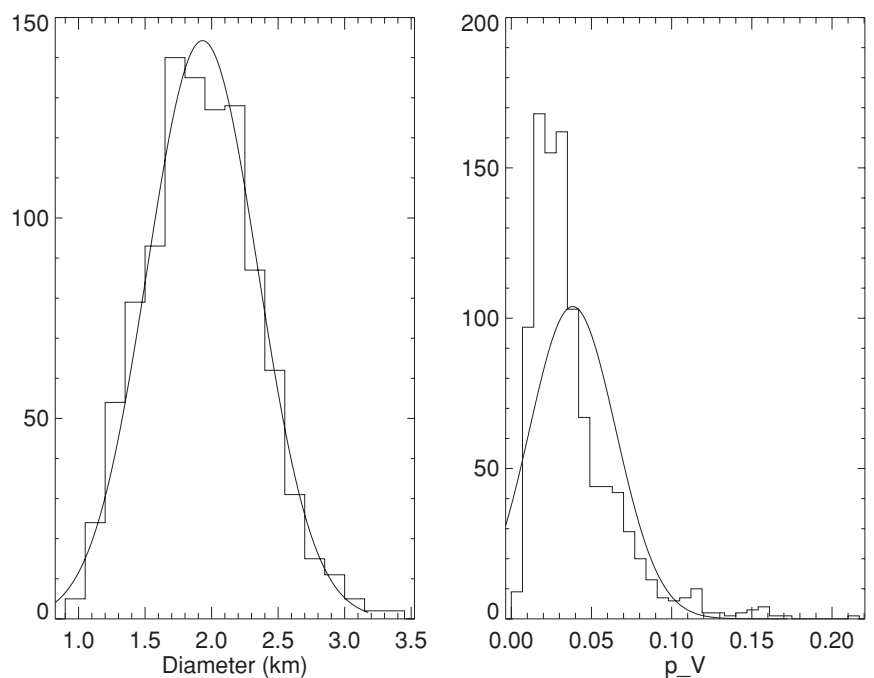

Figure 1. Histogram of the distribution of diameter and albedo values resulting from the Monte Carlo procedure for 2001 SK162. Both histograms are overplotted with the respective best-fit Gaussian functions. Note that the diameter distribution is rather consistent with being normally distributed, while the albedo distribution is clearly not.

the measured flux uncertainty, the calibration uncertainty of $5 \%$ (Carey et al. 2010), the uncertainty in $H$ (see below), and the NEATM temperature parameter $\eta$ (which is assumed to vary by \pm 0.3 around its nominal value; see below). For each observation, we generate 1000 sets of random synthetic fluxes normally distributed about the measured value and with a standard deviation equal to the root sum square of the measured flux uncertainty and the 5\% calibration uncertainty. Analogously, Gaussian distributions of $H$ and $\eta$ values are used in the fit. The distribution of albedo results (diameters to a lesser extent) is strongly non-Gaussian, see Figure 1 . We hence adopt the median of our Monte Carlo results as the nominal value and asymmetric error bars to encompass the central $68.2 \%$ of the results. Additionally, we determine the percentage of albedo results falling into albedo bins (see Section 4).

$\Delta H$. In the analysis of purely thermal observations of asteroids, $H$ is known to have a negligible influence on best-fit diameters but impacts $p_{\mathrm{V}}$ directly (Harris \& Harris 1997). In our case, however, $H$ is also used in correcting for reflected solar flux and hence influences the calculated thermal-flux contribution. We therefore decided to vary $H$ within the Monte Carlo fit. The correction for reflected sunlight becomes more critical as more reflected sunlight is contained within the measured flux; it is hence particularly important for high-albedo objects and for objects observed at large heliocentric distance.

Propagating $\Delta H$ into thermal-flux uncertainties is an update of the thermal-modeling pipeline relative to Trilling et al. (2010). While this does not change the calculated nominal thermal fluxes, it does change their uncertainties and hence the relative weight with which they enter the $\chi^{2}$ minimization procedure, leading to somewhat different diameter and albedo results. For practically all targets, however, the corresponding diameter change is $<1 \%$ and hence negligible.

For most of our targets, published values for $H$, let alone $\Delta H$, are unavailable. An observing campaign to measure $H$ for a number of our targets is currently underway; results will be reported in a separate paper. For the time being, we fall back to the approximate $H$ values given by the Horizons ephemeris server which are notoriously unreliable ${ }^{12}$ (see Jurić et al. 2002; Parker et al. 2008). For now, we adopt $\Delta H=0.5$ mag throughout, see Section 4.1 for exceptions.

$\Delta \eta$. Changes in the assumed $\eta$ value lead to significant changes in diameter and albedo, see, e.g., Harris et al. (2011). The quoted uncertainties in Equation (1) lead to a final $\Delta \eta \sim 0.3$ for $\alpha \sim 50^{\circ}$, a typical value for our sample. This uncertainty estimate is corroborated by Ryan \& Woodward (2010), who found a typical $\eta$ value of $1.07 \pm 0.27$; we caution, however, that their sample is dominated by large main-belt asteroids, whose thermal properties are rather distinct from our sample of small NEOs. For our Monte Carlo analysis, we therefore adopt a Gaussian distribution of $\eta$ values scattering about the nominal value of $0.91+0.013 \alpha$ with a standard deviation of 0.3 . Unphysical $\eta$ values below 0.5 are discarded.

\section{SIZES AND ALBEDOS}

Our diameter and albedo results are given in Table 2 along with their statistical uncertainties estimated from the Monte Carlo analysis described above. In order to illustrate the implications of our albedo results on surface mineralogy, we also determine the probabilities $p_{1}-p_{4}$ with which the albedo falls within one of the four albedo bins; these probabilities are estimated as the fraction of Monte Carlo albedos falling within the respective bin. The albedo bins are designed to correspond to taxonomic types as closely as possible, particularly for the purpose of identifying primitive NEOs.

1. $p_{\mathrm{V}}<7.5 \%$ : as shown by Fernández et al. (2005), albedos in this range strongly indicate a primitive surface composition.

2. $7.5 \% \leqslant p_{\mathrm{V}}<15 \%$ : while objects in this albedo range are still likely to be primitive, some of them may be more akin to (silicate-rich) $\mathrm{S}$ types.

3. $15 \% \leqslant p_{\mathrm{V}}<30 \%$ : objects in this albedo range are most likely $\mathrm{S}$ or $\mathrm{Q}$ types (or $\mathrm{M}$ types, but those are relatively rare among NEOs).

4. $30 \% \leqslant p_{\mathrm{V}}$ : more exotic compositions, e.g., E types.

\subsection{Reanalysis with Updated $H$ and $G$ Values for Select Objects}

In the analysis above, we assume $G=0.15$ and use $H$ magnitudes from the Horizons ephemeris service. While both assumptions are known to be problematic, we are constrained to use them in the "mass production" of diameters and albedo for practical reasons: we are not aware of a central database of published $H$ and $G$ values; rather, values for each target have to be searched for in the literature and are unavailable for a large majority. In order to minimize the induced uncertainties, we reanalyze our data for select objects with published $H$ and/or $G$ values; see Table 3 . We focus on objects which we find to have low albedo, as well as Eros and Itokawa. Where available, we also include published determinations of the size, albedo, or taxonomic type. The results of this reanalysis are given in Table 4.

Low-albedo objects. For (175706) 1996 FG3, (65679) 1989 UQ, and (100085) 1992 UY4, our albedo results are low, as expected based on their known taxonomic classification. In the case of 1996 FG3, there is also an excellent quantitative match between our results and the ground-based measurements quoted

\footnotetext{
12 It should not be forgotten that Horizons is not designed to calculate $H$
} magnitudes but ephemerides, at which it does an excellent job. 
Table 2

Results of our Spitzer Observations and of the Dynamical Analysis Described in Section 5

\begin{tabular}{|c|c|c|c|c|c|c|c|c|c|c|c|c|c|}
\hline $\begin{array}{l}\Delta v \\
\left(\mathrm{~km} \mathrm{~s}^{-1}\right)\end{array}$ & Object & $\begin{array}{c}D \\
(\mathrm{~km})\end{array}$ & $p_{\mathrm{V}}$ & $p_{1}$ & $p_{2}$ & $p_{3}$ & $p_{4}$ & $\begin{array}{l}q_{10 \%} \\
\text { (AU) }\end{array}$ & $\begin{array}{l}q_{50 \%} \\
\text { (AU) }\end{array}$ & $\begin{array}{l}q_{90 \%} \\
\text { (AU) }\end{array}$ & $\begin{array}{l}T_{10 \%} \\
(\mathrm{~K})\end{array}$ & $\begin{array}{c}T_{50 \%} \\
(\mathrm{~K})\end{array}$ & $\begin{array}{c}T_{90 \%} \\
(\mathrm{~K})\end{array}$ \\
\hline 4.632 & (25143) Itokawa & $0.319_{-0.050}^{+0.045}$ & $0.41_{-0.18}^{+0.20}$ & 0.003 & 0.017 & 0.277 & 0.703 & 0.376 & 0.737 & 0.887 & 644 & 460 & 419 \\
\hline 4.755 & 1996 XB27 & $0.084_{-0.012}^{+0.013}$ & $0.48_{-0.19}^{+0.26}$ & 0.000 & 0.017 & 0.167 & 0.817 & 0.612 & 0.658 & 1.000 & 501 & 483 & 392 \\
\hline 4.887 & (10302) $1989 \mathrm{ML}$ & $0.248_{-0.043}^{+0.035}$ & $0.47_{-0.19}^{+0.28}$ & 0.000 & 0.010 & 0.180 & 0.810 & 0.279 & 0.673 & 1.000 & 743 & 478 & 392 \\
\hline 5.276 & (99799) 2002 LJ3 & $0.503_{-0.085}^{+0.094}$ & $0.43_{-0.18}^{+0.22}$ & 0.000 & 0.033 & 0.233 & 0.733 & 0.313 & 0.665 & 0.875 & 704 & 483 & 421 \\
\hline 5.280 & $2001 \mathrm{CQ} 36$ & $0.068_{-0.012}^{+0.011}$ & $0.41_{-0.16}^{+0.29}$ & 0.000 & 0.030 & 0.223 & 0.747 & 0.145 & 0.579 & 1.000 & 1038 & 519 & 395 \\
\hline 5.302 & (52381) 1993 HA & $0.337_{-0.078}^{+0.097}$ & $0.140_{-0.077}^{+0.110}$ & 0.210 & 0.337 & 0.357 & 0.097 & 0.258 & 0.668 & 0.874 & 802 & 498 & 435 \\
\hline 5.328 & 2000 YF29 & $0.244_{-0.038}^{+0.041}$ & $0.251_{-0.095}^{+0.154}$ & 0.003 & 0.140 & 0.487 & 0.370 & 0.229 & 0.663 & 0.838 & 840 & 494 & 439 \\
\hline 5.391 & (1943) Anteros & $2.48_{-0.60}^{+0.69}$ & $0.145_{-0.073}^{+0.146}$ & 0.183 & 0.330 & 0.350 & 0.137 & 0.313 & 0.665 & 0.875 & 727 & 499 & 435 \\
\hline 5.486 & (138911) 2001 AE2 & $0.352_{-0.069}^{+0.073}$ & $0.34_{-0.16}^{+0.22}$ & 0.000 & 0.090 & 0.337 & 0.573 & 0.310 & 0.627 & 0.885 & 715 & 503 & 423 \\
\hline 5.487 & 2006 SY 5 & $0.090_{-0.017}^{+0.0013}$ & $0.34_{-0.14}^{+0.10}$ & 0.007 & 0.083 & 0.320 & 0.590 & 0.302 & 0.572 & 0.879 & 725 & 527 & 425 \\
\hline 5.555 & (162416) 2000 ЕH26 & $0.141_{-0.024}^{+0.026}$ & $0.181_{-0.073}^{+0.142}$ & 0.053 & 0.313 & 0.443 & 0.190 & 0.289 & 0.775 & 0.901 & 754 & 460 & 427 \\
\hline 5.565 & (162998) 2001 SK162 & $1.94_{-0.37}^{+0.38}$ & $0.031_{-0.015}^{+0.028}$ & 0.920 & 0.077 & 0.003 & 0.000 & 0.244 & 0.793 & 0.967 & 834 & 462 & 418 \\
\hline 5.653 & (68372) 2001 PM9 & $1.73_{-0.41}^{+0.45}$ & $0.0180_{-0.0080}^{+0.0170}$ & 0.970 & 0.030 & 0.000 & 0.000 & 0.278 & 0.711 & 0.872 & 782 & 488 & 441 \\
\hline 5.719 & (12923) Zephyr & $1.86_{-0.46}^{+0.45}$ & $0.21_{-0.11}^{+0.17}$ & 0.070 & 0.247 & 0.410 & 0.273 & 0.304 & 0.714 & 0.903 & 733 & 478 & 425 \\
\hline 6.041 & (85938) 1999 DJ4 & $0.478_{-0.082}^{+0.100}$ & $0.28_{-0.13}^{+0.23}$ & 0.017 & 0.140 & 0.393 & 0.450 & 0.303 & 0.690 & 0.882 & 729 & 482 & 427 \\
\hline 6.069 & (433) Eros & $30.7_{-8.3}^{+10.0}$ & $0.065_{-0.034}^{+0.075}$ & 0.560 & 0.303 & 0.117 & 0.020 & 0.320 & 0.580 & 0.742 & 724 & 538 & 476 \\
\hline 6.070 & 2000 GV147 & $0.502_{-0.072}^{+0.104}$ & $0.185_{-0.084}^{+0.124}$ & 0.063 & 0.300 & 0.460 & 0.177 & 0.263 & 0.577 & 0.820 & 791 & 534 & 447 \\
\hline 6.086 & (217807) 2000 XK44 & $0.73_{-0.13}^{+0.14}$ & $0.28_{-0.12}^{+0.18}$ & 0.013 & 0.130 & 0.387 & 0.470 & 0.319 & 0.617 & 0.925 & 711 & 510 & 417 \\
\hline 6.106 & $1993 \mathrm{RA}$ & $0.358_{-0.052}^{+0.063}$ & $0.40_{-0.16}^{+0.23}$ & 0.007 & 0.040 & 0.300 & 0.653 & 0.323 & 0.713 & 0.924 & 697 & 468 & 411 \\
\hline 6.130 & (177614) 2004 HK33 & $0.94_{-0.18}^{+0.17}$ & $0.189_{-0.081}^{+0.141}$ & 0.073 & 0.267 & 0.457 & 0.203 & 0.306 & 0.638 & 0.820 & 732 & 507 & 447 \\
\hline 6.191 & (192559) 1998 VO & $0.216_{-0.032}^{+0.032}$ & $0.30_{-0.13}^{+0.17}$ & 0.007 & 0.107 & 0.380 & 0.507 & 0.247 & 0.521 & 0.745 & 805 & 554 & 463 \\
\hline 6.196 & (212546) 2006 SV19 & $1.06_{-0.21}^{+0.23}$ & $0.129_{-0.058}^{+0.104}$ & 0.183 & 0.420 & 0.297 & 0.100 & 0.216 & 0.809 & 1.000 & 876 & 453 & 407 \\
\hline 6.236 & 2005 JA22 & $0.67_{-0.14}^{+0.17}$ & $0.164_{-0.078}^{+0.117}$ & 0.120 & 0.320 & 0.423 & 0.137 & 0.200 & 0.747 & 0.970 & 908 & 470 & 412 \\
\hline 6.240 & (1627) Ivar & $9.4_{-2.3}^{+3.9}$ & $0.094_{-0.051}^{+0.138}$ & 0.390 & 0.297 & 0.253 & 0.060 & 0.347 & 0.681 & 0.840 & 695 & 496 & 446 \\
\hline 6.279 & (87024) 2000 JS66 & $0.312_{-0.039}^{+0.059}$ & $0.63_{-0.23}^{+0.34}$ & 0.000 & 0.003 & 0.057 & 0.940 & 0.279 & 0.598 & 0.874 & 728 & 497 & 411 \\
\hline 6.323 & (22099) 2000 EX106 & $0.621_{-0.076}^{+0.109}$ & $0.29_{-0.12}^{+0.16}$ & 0.003 & 0.113 & 0.413 & 0.470 & 0.138 & 0.530 & 0.804 & 1080 & 550 & 446 \\
\hline 6.364 & 2003 SL5 & $0.337_{-0.053}^{+0.062}$ & $0.38_{-0.16}^{+0.22}$ & 0.003 & 0.027 & 0.350 & 0.620 & 0.269 & 1.000 & 1.000 & 764 & 396 & 396 \\
\hline 6.364 & (35107) $1991 \mathrm{VH}$ & $1.12_{-0.20}^{+0.23}$ & $0.27_{-0.12}^{+0.20}$ & 0.023 & 0.143 & 0.413 & 0.420 & 0.094 & 0.525 & 0.884 & 1311 & 554 & 427 \\
\hline 6.379 & (172974) 2005 YW55 & $0.342_{-0.059}^{+0.079}$ & $0.30_{-0.13}^{+0.24}$ & 0.000 & 0.103 & 0.383 & 0.513 & 0.269 & 0.901 & 1.000 & 772 & 421 & 400 \\
\hline 6.405 & (65679) 1989 UQ & $0.73_{-0.15}^{+0.18}$ & $0.060_{-0.028}^{+0.059}$ & 0.647 & 0.270 & 0.077 & 0.007 & 0.144 & 0.530 & 1.000 & 1082 & 564 & 410 \\
\hline 6.431 & (159402) 1999 AP10 & $1.20_{-0.17}^{+0.29}$ & $0.35_{-0.16}^{+0.23}$ & 0.007 & 0.057 & 0.337 & 0.600 & 0.148 & 0.728 & 0.933 & 1033 & 466 & 412 \\
\hline 6.491 & (143651) 2003 QO104 & $2.29_{-0.51}^{+0.54}$ & $0.137_{-0.061}^{+0.140}$ & 0.160 & 0.380 & 0.327 & 0.133 & 0.243 & 0.639 & 1.000 & 826 & 509 & 407 \\
\hline 6.507 & $1989 \mathrm{AZ}$ & $1.09_{-0.19}^{+0.20}$ & $0.025_{-0.011}^{+0.019}$ & 0.967 & 0.030 & 0.003 & 0.000 & 0.229 & 0.541 & 0.791 & 861 & 560 & 463 \\
\hline 6.512 & (218863) 2006 WO127 & $1.70_{-0.34}^{+0.40}$ & $0.21_{-0.11}^{+0.17}$ & 0.060 & 0.227 & 0.443 & 0.270 & 0.213 & 0.622 & 0.924 & 875 & 512 & 420 \\
\hline 6.516 & (140158) 2001 SX169 & $0.58_{-0.12}^{+0.16}$ & $0.26_{-0.11}^{+0.19}$ & 0.033 & 0.150 & 0.433 & 0.383 & 0.204 & 0.454 & 0.667 & 891 & 596 & 492 \\
\hline 6.526 & (100085) 1992 UY4 & $2.60_{-0.64}^{+0.70}$ & $0.020_{-0.010}^{+0.022}$ & 0.943 & 0.050 & 0.007 & 0.000 & 0.684 & 0.862 & 0.977 & 498 & 444 & 417 \\
\hline 6.534 & (85839) 1998 YO4 & $1.81_{-0.36}^{+0.42}$ & $0.174_{-0.086}^{+0.136}$ & 0.103 & 0.310 & 0.417 & 0.170 & 0.269 & 0.901 & 1.000 & 783 & 427 & 406 \\
\hline 6.539 & (68359) $2001 \mathrm{OZ13}$ & $0.62_{-0.12}^{+0.15}$ & $0.42_{-0.19}^{+0.27}$ & 0.007 & 0.047 & 0.240 & 0.707 & 0.209 & 0.625 & 0.873 & 864 & 499 & 422 \\
\hline 6.577 & (11398) 1998 YP11 & $1.73_{-0.40}^{+0.47}$ & $0.176_{-0.090}^{+0.185}$ & 0.097 & 0.317 & 0.343 & 0.243 & 0.400 & 0.754 & 1.000 & 641 & 467 & 405 \\
\hline 6.608 & (155334) 2006 DZ169 & $1.15_{-0.29}^{+0.32}$ & $0.195_{-0.095}^{+0.183}$ & 0.080 & 0.253 & 0.387 & 0.280 & 0.275 & 0.803 & 1.000 & 771 & 451 & 404 \\
\hline 6.609 & (175706) 1996 FG3 & $1.90_{-0.44}^{+0.52}$ & $0.026_{-0.012}^{+0.029}$ & 0.923 & 0.067 & 0.010 & 0.000 & 0.307 & 0.531 & 0.730 & 743 & 565 & 482 \\
\hline 6.610 & (52760) 1998 ML14 & $0.81_{-0.14}^{+0.16}$ & $0.27_{-0.11}^{+0.24}$ & 0.007 & 0.140 & 0.413 & 0.440 & 0.456 & 0.731 & 0.895 & 594 & 469 & 424 \\
\hline 6.628 & (138947) 2001 BA40 & $0.440_{-0.080}^{+0.0855}$ & $0.42_{-0.18}^{+0.26}$ & 0.003 & 0.053 & 0.213 & 0.730 & 0.205 & 0.484 & 0.723 & 871 & 567 & 464 \\
\hline 6.652 & 2002 QE7 & $0.320_{-0.056}^{+0.061}$ & $0.34_{-0.14}^{+0.20}$ & 0.010 & 0.073 & 0.337 & 0.580 & 0.318 & 0.598 & 0.829 & 707 & 515 & 437 \\
\hline 6.652 & (5626) $1991 \mathrm{FE}$ & $3.96_{-0.92}^{+1.22}$ & $0.152_{-0.081}^{+0.159}$ & 0.173 & 0.323 & 0.340 & 0.163 & 0.644 & 1.000 & 1.000 & 507 & 406 & 406 \\
\hline 6.661 & (66251) 1999 GJ2 & $0.90_{-0.19}^{+0.24}$ & $0.37_{-0.19}^{+0.30}$ & 0.013 & 0.067 & 0.307 & 0.613 & 0.065 & 0.556 & 0.878 & 1558 & 533 & 424 \\
\hline 6.692 & (85990) 1999 JV6 & $0.498_{-0.088}^{+0.134}$ & $0.076_{-0.035}^{+0.058}$ & 0.500 & 0.373 & 0.120 & 0.007 & 0.142 & 0.457 & 0.629 & 1086 & 606 & 516 \\
\hline 6.703 & 1998 SE36 & $0.343_{-0.072}^{+0.069}$ & $0.30_{-0.14}^{+0.22}$ & 0.027 & 0.127 & 0.347 & 0.500 & 0.209 & 0.348 & 0.666 & 875 & 678 & 490 \\
\hline 6.738 & (5645) $1990 \mathrm{SP}$ & $2.20_{-0.64}^{+0.74}$ & $0.062_{-0.034}^{+0.079}$ & 0.597 & 0.260 & 0.110 & 0.033 & 0.305 & 0.576 & 0.811 & 743 & 540 & 456 \\
\hline 6.747 & (3671) Dionysus & $0.89_{-0.11}^{+0.11}$ & $0.67_{-0.24}^{+0.37}$ & 0.000 & 0.000 & 0.060 & 0.940 & 0.243 & 0.639 & 1.000 & 776 & 479 & 382 \\
\hline 6.750 & 2002 HF8 & $0.71_{-0.15}^{+0.16}$ & $0.181_{-0.081}^{+0.138}$ & 0.090 & 0.300 & 0.410 & 0.200 & 0.759 & 1.000 & 1.000 & 465 & 405 & 405 \\
\hline 6.751 & (164202) $2004 \mathrm{EW}$ & $0.157_{-0.021}^{+0.024}$ & $0.36_{-0.15}^{+0.22}$ & 0.000 & 0.043 & 0.317 & 0.640 & 0.144 & 0.530 & 1.000 & 1048 & 546 & 397 \\
\hline 6.757 & 2002 UN3 & $0.310_{-0.047}^{+0.052}$ & $0.67_{-0.26}^{+0.35}$ & 0.000 & 0.000 & 0.070 & 0.930 & 0.609 & 0.912 & 1.000 & 490 & 400 & 382 \\
\hline
\end{tabular}


Table 2

(Continued)

\begin{tabular}{|c|c|c|c|c|c|c|c|c|c|c|c|c|c|}
\hline $\begin{array}{l}\Delta v \\
\left(\mathrm{~km} \mathrm{~s}^{-1}\right)\end{array}$ & Object & $\begin{array}{c}D \\
(\mathrm{~km}) \\
\end{array}$ & $p_{\mathrm{V}}$ & $p_{1}$ & $p_{2}$ & $p_{3}$ & $p_{4}$ & $\begin{array}{l}q_{10 \%} \\
(\mathrm{AU}) \\
\end{array}$ & $\begin{array}{l}q_{50 \%} \\
(\mathrm{AU}) \\
\end{array}$ & $\begin{array}{l}q_{90 \%} \\
(\mathrm{AU}) \\
\end{array}$ & $\begin{array}{l}T_{10 \%} \\
(\mathrm{~K}) \\
\end{array}$ & $\begin{array}{l}T_{50 \%} \\
(\mathrm{~K})\end{array}$ & $\begin{array}{c}T_{90 \%} \\
(\mathrm{~K}) \\
\end{array}$ \\
\hline 6.817 & (40329) $1999 \mathrm{ML}$ & $0.96_{-0.23}^{+0.23}$ & $0.154_{-0.069}^{+0.157}$ & 0.117 & 0.370 & 0.347 & 0.167 & 0.608 & 0.994 & 1.000 & 521 & 408 & 406 \\
\hline 6.830 & 2005 EJ & $0.230_{-0.038}^{+0.041}$ & $0.43_{-0.19}^{+0.24}$ & 0.000 & 0.033 & 0.243 & 0.723 & 0.318 & 0.598 & 0.829 & 700 & 510 & 43 \\
\hline 6.840 & (5646) $1990 \mathrm{TR}$ & $2.03_{-0.28}^{+0.52}$ & $0.65_{-0.28}^{+0.43}$ & 0.000 & 0.020 & 0.080 & 0.900 & 0.400 & 1.000 & 1.000 & 606 & 383 & 38 \\
\hline 6.963 & 2003 BT47 & $1.15_{-0.24}^{+0.31}$ & $0.147_{-0.074}^{+0.149}$ & 0.173 & 0.340 & 0.340 & 0.147 & 0.650 & 0.901 & 1.000 & 504 & 428 & 406 \\
\hline 6.963 & (10115) 1992 SK & $0.90_{-0.18}^{+0.20}$ & $0.34_{-0.13}^{+0.25}$ & 0.003 & 0.050 & 0.360 & 0.587 & 0.129 & 0.491 & 0.754 & 1109 & 568 & 45 \\
\hline 6.974 & (5620) Jasonwheeler & $1.77_{-0.40}^{+0.46}$ & $0.094_{-0.046}^{+0.096}$ & 0.360 & 0.373 & 0.223 & 0.043 & 0.400 & 1.000 & 1.000 & 647 & 409 & 40 \\
\hline 6.981 & (152563) $1992 \mathrm{BF}$ & $0.51_{-0.11}^{+0.12}$ & $0.084_{-0.037}^{+0.077}$ & 0.410 & 0.407 & 0.147 & 0.037 & 0.242 & 0.514 & 0.686 & 833 & 571 & 49 \\
\hline 6.994 & (138971) 2001 CB21 & $0.578_{-0.079}^{+0.109}$ & $0.24_{-0.10}^{+0.12}$ & 0.020 & 0.213 & 0.487 & 0.280 & 0.142 & 0.457 & 0.629 & 1068 & 596 & 50 \\
\hline
\end{tabular}

Notes. $p_{1}-p_{4}$ denote the probability of the albedo falling within each of the four albedo bins described in the text (bin boundaries are 0.075 , 0.15 , and 0.3 ; primitive objects should display a high $p_{1}$ ). In the last six columns, $q_{x x}$ and $T_{x x}$ describe the orbital and thermal history (see Section 5). For example, $q_{10 \%}$ denotes the minimum perihelion distance that the object reached to within a probability of $10 \%$ and $T_{10 \%}$ is the corresponding temperature to which the surface was heated. Note that some of the diameter and albedo results herein are superseded in Table 4.

Table 3

Published Physical Properties for Objects Considered in Section 4.1

\begin{tabular}{|c|c|c|c|c|c|c|c|}
\hline Object & $H$ & $G$ & $D(\mathrm{~km})$ & $p_{\mathrm{V}}$ & $\operatorname{Bin}^{\mathrm{a}}$ & Taxo $^{b}$ & Reference \\
\hline (433) $\operatorname{Eros}^{c}$ & $10.46 \pm 0.10$ & 0.18 & $23.6^{\mathrm{a}}$ & $0.22^{\mathrm{b}}$ & $\ldots$ & $\mathrm{S}$ & 1,2 \\
\hline (25143) Itokawa & $19.51_{-0.08}^{+0.09 \mathrm{c}}$ & $0.29_{-0.06}^{+0.07}$ & $0.327 \pm 0.006$ & $0.26 \pm 0.02$ & $\ldots$ & $\mathrm{S}(\mathrm{IV})$ & 3,4 \\
\hline (10302) $1989 \mathrm{ML}$ & $\cdots$ & $\cdots$ & $0.28 \pm 0.05$ & $0.37 \pm 0.15$ & $\cdots$ & E & 5 \\
\hline (175706) 1996 FG3 & $17.76 \pm 0.03$ & -0.07 & $\sim 1.9$ & $\sim 0.04$ & $\mathrm{Y}$ & $\mathrm{C}$ & $6,7,8$ \\
\hline (1943) Anteros & $15.9 \pm 0.2$ & 0.23 & $\sim 2.2$ & $\sim 0.16$ & $\ldots$ & $\mathrm{L}$ & $9,10,11$ \\
\hline (65679) 1989 UQ & $19.5 \pm 0.3$ & $\cdots$ & $\cdots$ & $\cdots$ & $\cdots$ & B & 11,12 \\
\hline (162998) 2001 SK162 & & $\cdots$ & $\cdots$ & $\cdots$ & $\cdots$ & $\mathrm{T}$ & 11 \\
\hline (100085) 1992 UY4 & $17.71 \pm 0.10$ & $\cdots$ & 1.7 & 0.05 & $\cdots$ & $\mathrm{P}$ & 13,14 \\
\hline (152563) $1992 \mathrm{BF}$ & $\ldots$ & $\cdots$ & $\cdots$ & $\cdots$ & $\cdots$ & $X_{c}$ & 15 \\
\hline (12923) Zephyr & $\ldots$ & $\cdots$ & $\ldots$ & $\ldots$ & $\cdots$ & $\mathrm{S}$ & 16 \\
\hline (1627) Ivar & $12.87 \pm 0.10$ & $\cdots$ & $9.1 \pm 1.4$ & $0.15 \pm 0.05$ & $\cdots$ & $\mathrm{S}$ & 16,17 \\
\hline (85990) 1999 JV6 & $\ldots$ & $\ldots$ & $\ldots$ & $\ldots$ & $\ldots$ & $X_{k}$ & 15 \\
\hline (3671) Dionysus & $16.66 \pm 0.30$ & $\cdots$ & $1.1-1.5$ & $0.16-0.31$ & $\mathrm{Y}$ & $C_{b}$ & $15,18,19$ \\
\hline
\end{tabular}

Notes.

${ }^{a} \mathrm{Y}$ indicates that the object is known to be binary.

b Taxonomic type.

${ }^{c}$ See Trilling et al. (2010) for a discussion of the values adopted for Eros, which was observed at a nearly pole-on viewing geometry and Mueller (2007) for a discussion of its $G$ value.

References. (1) Harris \& Davies 1999; (2) Li et al. 2004; (3) Bernardi et al. 2008; (4) volume-equivalent diameter calculated from the volume given by Demura et al. 2006; (5) Mueller et al. 2007; (6) Pravec et al. 2006; (7) M. Mueller et al. (in preparation); (8) Binzel et al. 2001; (9) adopted after $15.82 \pm 0.14$ (Wisniewski et al. 1997) and $15.96 \pm 0.14$ (Pravec et al. 1998), both give $G=0.23$; (10) quoted after Harris et al. 2011, original data from Veeder et al. 1989; (11) Binzel et al. 2004a; (12) Pravec et al. 1998; (13) Warner et al. 2006-no $H$ uncertainty is given by Warner et al., $0.1 \mathrm{mag}$ seems appropriate (or slightly conservative) given the low scatter of their data; (14) Volquardsen et al. 2007—note that their error bars (not quoted herein) reflect only the statistical uncertainties, but not the systematics; (15) Bus \& Binzel 2002; (16) Binzel et al. 2004b; (17) Delbo' et al. 2003 determined $D$ and $p_{\mathrm{V}}$ of Ivar based on Keck mid-IR photometry, we here assume a 15\% uncertainty in $D$ and $30 \%$ in $p_{\mathrm{V}}$ as is usual for radiometric diameters. $H$ is from P. Pravec et al. (unpublished), quoted after Delbo' et al. 2003; (18) different, model-dependent, diameter and albedo values are given by Harris \& Davies 1999 and Harris \& Lagerros 2002—we quote the range of their adopted results. Harris \& Davies 1999 quote P. Pravec (unpublished) for the $H$ given herein, no uncertainty value is stated, we assume a conservative uncertainty of $0.3 \mathrm{mag}$; (19) Mottola et al. 1997.

in Table 3. Our diameter result for 1992 UY4 is formally in agreement with that by Volquardsen et al. (2007), provided that their value is assigned a realistic uncertainty of $\sim 15 \%$ to include systematic uncertainties (which are not discussed by the authors of that paper).

"Reality checks." While the general agreement between ExploreNEOs results and other published diameter and albedo results is discussed by Harris et al. (2011), we consider it useful to check some of our results for low- $\Delta v$ objects against published values. By comparing Tables 3 and 4, we find excellent agreement in the cases of (433) Eros, (25143) Itokawa, (10302) 1989 ML, (1943) Anteros, and (1627) Ivar.

(3671) Dionysus. Our nominal albedo result for Dionysus, $p_{\mathrm{V}}=0.55_{-0.17}^{+0.21}$, is hard to reconcile with its taxonomic 
Table 4

Reanalysis of Thermal Data with Updated $H$ and $G$ Values (see Table 3)

\begin{tabular}{|c|c|c|c|c|c|c|c|c|}
\hline Object & $D(\mathrm{~km})$ & $p_{\mathrm{V}}$ & $p_{1}$ & $p_{2}$ & $p_{3}$ & $p_{4}$ & $D^{*}$ & $p_{\mathrm{V}}{ }^{*}$ \\
\hline (433) Eros & $23.0_{-5.6}^{+8.3}$ & $0.218_{-0.099}^{+0.173}$ & 0.030 & 0.250 & 0.423 & 0.297 & $30.7_{-8.3}^{+10.0}$ & $0.065_{-0.034}^{+0.075}$ \\
\hline (25143) Itokawa & $0.313_{-0.044}^{+0.054}$ & $0.283_{-0.075}^{+0.116}$ & 0.000 & 0.023 & 0.550 & 0.427 & $0.319_{-0.050}^{+0.045}$ & $0.41_{-0.18}^{+0.20}$ \\
\hline (10302) $1989 \mathrm{ML}$ & $0.240_{-0.038}^{+0.043}$ & $0.50_{-0.18}^{+0.21}$ & 0.003 & 0.010 & 0.127 & 0.860 & $0.248_{-0.043}^{+0.035}$ & $0.47_{-0.19}^{+0.28}$ \\
\hline (175706) 1996 FG3 & $1.84_{-0.47}^{+0.56}$ & $0.042_{-0.017}^{+0.035}$ & 0.837 & 0.140 & 0.023 & 0.000 & $1.90_{-0.44}^{+0.52}$ & $0.026_{-0.012}^{+0.029}$ \\
\hline (1943) Anteros & $2.38_{-0.59}^{+0.72}$ & $0.138_{-0.061}^{+0.107}$ & 0.150 & 0.420 & 0.367 & 0.063 & $2.48_{-0.60}^{+0.69}$ & $0.145_{-0.073}^{+0.146}$ \\
\hline (65679) 1989 UQ & $0.72_{-0.14}^{+0.18}$ & $0.053_{-0.021}^{+0.036}$ & 0.753 & 0.223 & 0.023 & 0.000 & $0.73_{-0.15}^{+0.18}$ & $0.060_{-0.028}^{+0.059}$ \\
\hline (100085) 1992 UY4 & $2.50_{-0.58}^{+0.67}$ & $0.0230_{-0.0090}^{+0.0190}$ & 0.977 & 0.020 & 0.003 & 0.000 & $2.60_{-0.64}^{+0.70}$ & $0.020_{-0.010}^{+0.022}$ \\
\hline (1627) Ivar & $9.9_{-2.8}^{+2.8}$ & $0.128_{-0.052}^{+0.123}$ & 0.157 & 0.447 & 0.273 & 0.123 & $9.4_{-2.3}^{+3.9}$ & $0.094_{-0.051}^{+0.138}$ \\
\hline (3671) Dionysus & $0.86_{-0.11}^{+0.12}$ & $0.55_{-0.17}^{+0.21}$ & 0.000 & 0.000 & 0.047 & 0.953 & $0.89_{-0.11}^{+0.11}$ & $0.67_{-0.24}^{+0.37}$ \\
\hline Dionysus with $\eta=3$ & $1.46_{-0.18}^{+0.21}$ & $0.179_{-0.065}^{+0.092}$ & 0.020 & 0.320 & 0.553 & 0.107 & $\ldots$ & $\ldots$ \\
\hline
\end{tabular}

Notes. In the last two columns, the diameter and albedo results for the "nominal" $H$ and $G$ values are given for comparison (see Table 2 ). In this reanalysis, $\eta=1.07$ is assumed for (433) Eros (see Trilling et al. 2010). Two $\eta$ assumptions are made for (3671) Dionysus, the one used in the rest of this manuscript (see Equation (1); upper line), and $\eta=3$ (lower line). For all objects except Eros and Dionysus, $D$ and $D^{*}$ values are practically indistinguishable, implying that diameter results are not significantly impacted by the $H$ uncertainty.

classification as $C_{b}$ type, for which a low albedo would be expected. Harris \& Davies (1999) report UKIRT mid-IR observations of Dionysus from which they derive $p_{\mathrm{V}}=0.35$ or $p_{\mathrm{V}}=0.61$, depending on thermal model. Due to "inadequate signal to noise," they were unable to constrain $\eta$ from their data, like in our case. However, Harris \& Davies also report Infrared Space Observatory (ISO) data, from which $\eta$ can be constrained (if marginally so) to be $\sim 3.1$, in the upper range of plausible $\eta$ values. This results in $p_{\mathrm{V}}=0.16$ (quoted after Harris \& Lagerros 2002-Harris \& Davies reject that result in favor of another thermal model, leading to much higher albedo). We have therefore repeated the analysis of our Dionysus data assuming $\eta=3$ (the phase angle of our observations, $\alpha=62.6$, is very similar to that of the ISO observations of Harris \& Davies, 57.7). The resulting albedo, $p_{\mathrm{V}}=0.178_{-0.065}^{+0.092}$, is in good agreement with Harris' NEATM result $\left(p_{\mathrm{V}}=0.16\right)$ and consistent with a $C_{b}$ classification given the error bars.

We note that binary NEOs including Dionysus were recently found to generally display higher-than-average $\eta$ values (Delbo' et al. 2011), probably due to regolith loss during binary formation. This may be expected to reduce the accuracy of our results for binaries in general. However, in the case of the only other known low-albedo binary, 1996 FG3 (see above), our results are quite consistent with those obtained otherwise.

\section{THERMAL HISTORY}

It is well known that implantation of solar wind ions (Hapke 2001) and bombardment by micrometeorites can alter the spectroscopic properties of asteroids (Sasaki et al. 2001). However, these aging processes affect only the topmost microns of the surface. This is not a problem for a sample collection experiment: current-technology sampling devices can sample material from a depth of a few centimeters, thus excavating below the space-weathered surface.

However, Marchi et al. (2009) have shown that the surfaces of a significant number of NEOs were heated by the Sun to very high temperatures that could induce surface alterations on previously primitive objects. Due to thermal conduction, a thin layer beneath the surface can be heated to similarly large temperatures; for typical thermal properties (Mueller 2007; Delbo' et al. 2007) and rotation periods, the penetration depth of the heat wave is of the order of centimeters (Spencer et al. 1989), comparable to the digging depth of sample-taking devices.
There is no clear-cut way to determine the maximum temperature to which an asteroid can be heated and remain in primitive condition. From laboratory studies of carbonaceous chondrite meteorites, to which primitive asteroids are generally believed to be related, we know different alteration processes that are characterized by different threshold temperatures (e.g., thermal breakup of organic macromolecules). For instance, it has been determined by laboratory heating experiments that at $370 \mathrm{~K}$ the insoluble organic matter of the carbonaceous meteorite Murchison is degraded (the aliphatic $\mathrm{C}-\mathrm{H}$ bond is lost) in approximately 200 years (Kebukawa et al. 2010). The same authors also showed that the bulk organics of Murchison are lost in only one year at $370 \mathrm{~K}$ (or 200 years at $300 \mathrm{~K}$ ). It is also known that the macromolecular phase in carbonaceous meteorites has a structure similar to refractory kerogen. The latter starts to break up - with production of oil and gas - when heated above 420 K (I. Franchi 2008, private communication). Furthermore, Lauretta et al. (2001) have shown experimentally that volatile components (such as $\mathrm{Hg}$ ) are released from some $\mathrm{CM}$ and $\mathrm{CV}$ carbonaceous chondrites when the latter are heated above $\sim 470 \mathrm{~K}$.

The maximum temperature attained by an NEO is a function of the perihelion distance $q$. NEO orbits can evolve rapidly (see, e.g., Michel \& Froeschlé 1997), hence the present $q$ is not particularly indicative of the minimum $q$ attained within the chaotic dynamical history. Hence, if it is a goal to send a spacecraft to a primitive object, the dynamical and thermal history of the target must be taken into account.

An upper limit on the subsurface temperature is the surface temperature at local noon (assuming equilibrium with absorbed insolation). Because spin axes of NEOs evolve on relatively short timescales (e.g., due to YORP, planetary encounters, and possibly due to spin-orbit coupling), the whole surface is likely to have been subjected to temperatures (nearly) as high as that of the subsolar point, $T_{\mathrm{SS}}$. We calculate $T_{\mathrm{SS}}$ as a function of $q$ using the NEATM (see Section 3), assuming the nominal albedo resulting from our Spitzer observations and $\eta=0.91$. That latter value follows from Equation (1) for $\alpha=0$, hence providing an upper limit on temperature.

With this in mind, the maximum surface temperature attained follows from the minimum $q$ reached in the past. Due to the chaotic nature of NEO orbits, this question must be treated probabilistically. We here use the orbital evolution model by 
Table 5

Potentially Primitive Objects as Indicated by their Low Albedo $\left(p_{1}>50 \%\right)$

\begin{tabular}{|c|c|c|c|c|c|c|c|c|}
\hline $\begin{array}{l}\Delta v \\
\left(\mathrm{~km} \mathrm{~s}^{-1}\right)\end{array}$ & Object & $p_{1}$ & $\begin{array}{c}D \\
(\mathrm{~km})\end{array}$ & $p_{\mathrm{V}}$ & $\begin{array}{c}T_{10 \%} \\
(\mathrm{~K})\end{array}$ & $\begin{array}{c}T_{50 \%} \\
(\mathrm{~K})\end{array}$ & $\begin{array}{c}T_{90 \%} \\
(\mathrm{~K})\end{array}$ & Taxo \\
\hline 5.565 & (162998) 2001 SK162 & 0.92 & $1.9 \pm 0.4$ & $0.03_{-0.02}^{+0.03}$ & 834 & 462 & 418 & $\mathrm{~T}$ \\
\hline 5.653 & (68372) 2001 PM9 & 0.97 & $1.7 \pm 0.5$ & $0.018_{-0.008}^{+0.017}$ & 782 & 488 & 441 & $\cdots$ \\
\hline 6.405 & (65679) 1989 UQ & 0.75 & $0.7 \pm 0.2$ & $0.05_{-0.02}^{+0.04}$ & 1082 & 564 & 410 & $\mathrm{~B}$ \\
\hline 6.507 & $1989 \mathrm{AZ}$ & 0.97 & $1.1 \pm 0.2$ & $0.03_{-0.01}^{+0.02}$ & 861 & 560 & 463 & $\cdots$ \\
\hline 6.526 & (100085) 1992 UY4 & 0.98 & $2.5_{-0.6}^{+0.7}$ & $0.023_{-0.009}^{+0.019}$ & 498 & 444 & 417 & $\mathrm{P}$ \\
\hline 6.609 & (175706) 1996 FG3 & 0.84 & $1.8_{-0.5}^{+0.6}$ & $0.04_{-0.02}^{+0.04}$ & 743 & 565 & 482 & $\mathrm{C}$ \\
\hline 6.738 & (5645) $1990 \mathrm{SP}$ & 0.60 & $2.2_{-0.7}^{+0.8}$ & $0.06_{-0.04}^{+0.08}$ & 743 & 540 & 456 & $\ldots$ \\
\hline
\end{tabular}

Notes. See Table 2 for the definition of $p_{1}$. Where available, diameter and albedo results are from the reanalysis in Table 4 , otherwise from Table 2. As discussed in Section 5, the chances are 10\%/50\%/90\% that the surface temperature has reached $T_{10 \% / 50 \% / 90 \%}$ or above.

Marchi et al. (2009), which was derived from Bottke et al. $(2000,2002)$. For each of our targets, we extract from the work by Marchi et al. the probabilities that the object reached a perihelion distance smaller than a grid of $q$ values. Through interpolation, we determine $q_{50 \%}$, i.e., the $q$ value which was reached with a probability of $50 \%$. We call the corresponding subsolar temperature $T_{50 \%}$. The odds are $50 \%$ that an object ventured to within $q_{50 \%}$ of the Sun and that its surface was heated to $T_{50 \%}$ or more. We repeat this exercise for probability values of $10 \%$ and $90 \%$; results are given in Table 2 .

Note, for instance, that (65679) 1989 UQ certainly appears primitive judging from its low albedo (this work) and its spectroscopic classification as B type (Binzel et al. 2004a). 1989 UQ was considered repeatedly as a target of sample-return mission concepts to a primitive asteroid. However, we show that this object has likely been heated to the point that its organic macromolecular matter has been broken up $\left(T_{50 \%}=564 \mathrm{~K}-\mathrm{cf}\right.$. Table 2). The same applies to the binary system (175706) 1996 FG3.

However, the final determination whether an object is to be considered primitive or not is beyond our scope. Rather, our aim is to provide the required input data for that determination. Given that objects may have exceeded some but not all of the threshold temperatures discussed above, the exact definition of "primitive" depends on the scientific purpose at hand. Also, it is incumbent on the mission-design team to quantify the risk they are willing to take (note the probabilistic nature of our temperature determinations due to the chaotic orbital history of NEOs).

\section{DISCUSSION}

\subsection{Updated ExploreNEOs Thermal-modeling Pipeline}

For a fraction of our targets, our data have been published previously along with a straightforward NEATM analysis (Trilling et al. 2010; Harris et al. 2011). Due to the updates in the thermalmodeling pipeline presented in Section 3.1, our results given in Table 2 supersede previous values where available. This difference, however, is always comfortably within the quoted error bars and too small to matter practically. A new analysis of the entire data set including new observations will be presented in a later paper.

The new pipeline provides estimates of the statistical uncertainty of diameter and albedo results. The uncertainties are distributed in a highly non-Gaussian way, especially for $p_{\mathrm{V}}$, hence asymmetric " $1 \sigma$ " error bars are given. Additionally, we provide probabilities of $p_{\mathrm{V}}$ to fall into specific bins, which allows for more straightforward constraints on the taxonomic type and hence surface mineralogy.

\subsection{Potential Spacecraft Targets}

1996 XB27 and 1989 ML. These bodies are, along with the Hayabusa target Itokawa, the only objects with $\Delta v<5 \mathrm{~km} \mathrm{~s}^{-1}$ within our sample. We find both objects to be very high in albedo, indicative of taxonomic types such as E (1989 ML was found to be E type by Mueller et al. 2007). The chances of them being primitive are very small. With a diameter of only $84_{-12}^{+13} \mathrm{M}$, 1996 XB27 is among the smallest celestial objects with known size.

Primitive objects. Some of our albedo results suggest a primitive composition. We adopt a threshold value of $50 \%$ for $p_{1}$, the probability of $p_{\mathrm{V}}<7.5 \%$. A list of all measured physical properties of these objects is compiled in Table 5. There are significant object-to-object differences in thermal history. As discussed in Section 5, there is no clearly defined threshold temperature above which primitive material is metamorphosed. The least heated objects (at the 50\% probability level) are 1992 UY4, 2001 SK162, and 2001 PM9. 1996 FG3 is the only known binary in the low- $p_{\mathrm{V}}$ sample.

\section{CONCLUSIONS}

Of the 293 NEOs observed within the framework of our ongoing ExploreNEOs survey as of 2010 July 14, 65 have $\Delta v \leqslant 7 \mathrm{~km} \mathrm{~s}^{-1}$. Diameter and albedo measurements for the latter are presented in this work. Assuming that the rate of observations of low- $\Delta v$ objects stays as it is, the number of observed low- $\Delta v$ objects will increase to $\sim 160$ by the end of ExploreNEOs, i.e., before the end of 2011. Teams requiring a physical characterization of potential spacecraft targets are encouraged to contact us.

Out of our 65 low- $\Delta v$ targets, 7 have low albedos indicating a primitive surface composition. These objects include a binary (1996 FG3) and three objects which stayed remarkably cool during their dynamical history, possibly cool enough to remain primitive: 1992 UY4, 2001 SK162, and 2001 PM9.

Michael Mueller gratefully acknowledges the Henri Poincaré Fellowship, which is funded by the CNRS-INSU and the Conseil Général des Alpes-Maritimes. The work of M.M. and M.D. is supported by ESA grant SSA-NEO-ESA-MEM-017/1. We thank Patrick Michel for helpful discussions. This work is based 
in part on observations made with the Spitzer Space Telescope, which is operated by JPL/Caltech under a contract with NASA. Support for this work was provided by NASA through an award issued by JPL/Caltech.

Facilities: Spitzer(IRAC)

\section{REFERENCES}

Abell, P. A., et al. 2009, Meteorit. Planet. Sci., 44, 1825

Bernardi, F., Tholen, D. J., \& Micheli, M. 2008, ACM Meeting 2008, http://www.lpi.usra.edu/meetings/acm2008/pdf/8338.pdf

Binzel, R. P., Harris, A. W., Bus, S. J., \& Burbine, T. H. 2001, Icarus, 151, 139

Binzel, R. P., Perozzi, E., Rivkin, A. S., Rossi, A., Harris, A. W., Bus, S. J., Valsecchi, G. B., \& Slivan, S. M. 2004a, Meteorit. Planet. Sci., 39, 351

Binzel, R. P., Rivkin, A. S., Stuart, J. S., Harris, A. W., Bus, S. J., \& Burbine, T. H. 2004b, Icarus, 170, 259

Bottke, W. F., Jedicke, R., Morbidelli, A., Petit, J.-M., \& Gladman, B. 2000, Science, 288, 2190

Bottke, W. F., Morbidelli, A., Jedicke, R., Petit, J.-M., Levison, H. F., Michel, P., \& Metcalfe, T. S. 2002, Icarus, 156, 399

Bus, S. J., \& Binzel, R. P. 2002, Icarus, 158, 146

Carey, S. J., et al. 2010, Proc. SPIE, 7731, 77310N

Delbo', M., dell'Oro, A., Harris, A. W., Mottola, S., \& Mueller, M. 2007, Icarus, 190,236

Delbo', M., Harris, A. W., Binzel, R. P., Pravec, P., \& Davies, J. K. 2003, Icarus, 166,116

Delbo', M., Walsh, K., Mueller, M., Harris, A. W., \& Howell, E. S. 2011, Icarus, in press

Demura, H., et al. 2006, Science, 312, 1347

Fazio, G. G., et al. 2004, ApJS, 154, 10

Fernández, Y. R., Jewitt, D. C., \& Sheppard, S. S. 2005, AJ, 130, 308

Hahn, G., et al. 1989, Icarus, 78, 363

Hapke, B. 2001, J. Geophys. Res., 106, 10039

Harris, A. W. 1998, Icarus, 131, 291

Harris, A. W., \& Davies, J. K. 1999, Icarus, 142, 464

Harris, A. W., \& Harris, A. W. 1997, Icarus, 126, 450

Harris, A. W., \& Lagerros, J. S. V. 2002, in Asteroids III, ed. W. F. Bottke, A. Cellino, P. Paolicchi, \& R. P. Binzel (Tucson, AZ: Univ. Arizona Press), 205

Harris, A. W., Mueller, M., Lisse, C. M., \& Cheng, A. F. 2009, Icarus, 199, 86
Harris, A. W., et al. 2011, AJ, 141, 75

Jurić, M., et al. 2002, AJ, 124, 1776

Kebukawa, Y., Nakashima, S., \& Zolensky, M. E. 2010, Meteorit. Planet. Sci., 45, 99

Lauretta, D. S., Klaue, B., Blum, J. D., \& Buseck, P. R. 2001, in Lunar and Planetary Institute Science Conference Abstracts, Vol. 32, ed. LPI Ed. Board (Houston: LPI), 1356

Lauretta, D. S., et al. 2010, 73rd Annual Meteoritical Society Meeting, http://www.lpi.usra.edu/meetings/metsoc2010/pdf/5153.pdf

Li, J., A'Hearn, M. F., \& McFadden, L. A. 2004, Icarus, 172, 415

Marchi, S., Delbo', M., Morbidelli, A., Paolicchi, P., \& Lazzarin, M. 2009, MNRAS, 400, 147

Michel, P., \& Froeschlé, C. 1997, Icarus, 128, 230

Michel, P., et al. 2009, 72nd Annual Meeting of the Meteoritical Society, http://www.lpi.usra.edu/meetings/metsoc2009/pdf/5261.pdf

Mottola, S., Hahn, G., Pravec, P., \& Šarounová, L. 1997, IAU Circ., 6680

Mueller, M. 2007, PhD thesis, Freie Univ., Berlin, http://www.diss.fu-berlin.de/ 2007/471/indexe.htm

Mueller, M., Harris, A. W., \& Fitzsimmons, A. 2007, Icarus, 187, 611

Okada, T., et al. 2010, 41st LPSC Meeting, http://www.lpi.usra.edu/meetings/ lpsc2010/pdf/2132.pdf

Parker, A., Ivezić, Ž., Jurić, M., Lupton, R., Sekora, M. D., \& Kowalski, A. 2008, Icarus, 198, 138

Pravec, P., Wolf, M., \& Šarounová, L. 1998, Icarus, 136, 124

Pravec, P., et al. 2006, Icarus, 181, 63

Ryan, E. L., \& Woodward, C. E. 2010, AJ, 140, 933

Sasaki, S., Nakamura, K., Hamabe, Y., Kurahashi, E., \& Hiroi, T. 2001, Nature, 410,555

Shoemaker, G., \& Helin, E. 1978, NASA CP-2053, 245, http://ntrs.nasa.gov/ archive/nasa/casi.ntrs.nasa.gov/19780021079_1978021079.pdf

Spencer, J. R., Lebofsky, L. A., \& Sykes, M. V. 1989, Icarus, 78, 337

Trilling, D. E., et al. 2008, ApJ, 683, L199

Trilling, D. E., et al. 2010, AJ, 140, 770 (ExploreNEOs I)

Veeder, G. J., Hanner, M. S., Matson, D. L., Tedesco, E. F., Lebofsky, L. A., \& Tokunaga, A. T. 1989, AJ, 97, 1211

Volquardsen, E. L., Rivkin, A. S., \& Bus, S. J. 2007, Icarus, 187, 464

Warner, B. D., et al. 2006, Minor Planet Bull., 33, 20

Werner, M. W., et al. 2004, ApJS, 154, 1

Wisniewski, W. Z., Michałowski, T. M., Harris, A. W., \& McMillan, R. S. 1997, Icarus, 126, 395

Wolters, S. D., Green, S. F., McBride, N., \& Davies, J. K. 2008, Icarus, 193, 535 